Research Article

\title{
Finite-Element Analysis of Keying Process of Plate Anchors in Three-Layer Soft-Stiff-Soft Clay Deposits
}

\author{
Tugen Feng, ${ }^{1,2}$ Huajiao Xu, ${ }^{3}$ Jian Song $\mathbb{D}^{1,2}$ Jian Zhang, ${ }^{1,2}$ Mi Zhou, ${ }^{4}$ and Fuhai Zhang ${ }^{1,2}$ \\ ${ }^{1}$ Key Laboratory of Ministry of Education for Geomechanics and Embankment Engineering, Hohai University, \\ Nanjing 210098, China \\ ${ }^{2}$ Geotechnical Research Institute, Hohai University, Nanjing 210098, China \\ ${ }^{3}$ Jiang Run Technology Co., Ltd., Hangzhou 310000, China \\ ${ }^{4}$ State Key Laboratory of Subtropical Building Science, South China Institute of Geotechnical Engineering, \\ South China University of Technology, Guangzhou 510640, China
}

Correspondence should be addressed to Jian Song; jiansonghh@163.com

Received 22 February 2019; Revised 6 June 2019; Accepted 23 June 2019; Published 7 July 2019

Academic Editor: Giovanni Garcea

Copyright (c) 2019 Tugen Feng et al. This is an open access article distributed under the Creative Commons Attribution License, which permits unrestricted use, distribution, and reproduction in any medium, provided the original work is properly cited.

\begin{abstract}
This paper presents the results from numerical modeling of the keying process of plate anchors in three-layer soft-stiff-soft clay deposits. Three-dimensional large deformation finite-element analyses were carried out, and the results were firstly validated by the centrifuge test data and the previous numerical results. The soil flow mechanism during the keying process of plate anchors was examined, and a series of parametric studies were performed to investigate the factors affecting the rotation characteristics of plate anchors with an emphasis on the presence of the interbedded stiff soil layer. The results indicate that the loss of embedment depth of plate anchors decreases with the increase of the thickness of the first soil layer when the anchor is initially located at the middle of stiff soil layer. The flow velocity of soil around the anchor that is initially embedded at the first layer and adjacent to the underlying interbedded stiff soil layer is generally larger, resulting in a smaller embedment depth loss compared with the traditional normally consolidated soil layer. The interbedded stiff soil layer affects the keying process of plate anchors embedded $1.0 \mathrm{~B}$ above and $2.0 \mathrm{~B}$ below the interbedded stiff soil layer $(B$ is the width of the square plate anchors). The increase of the strength of local soil around the plate anchors leads to the increase of the embedment depth loss, but the increase of the strength of soil slightly away from the plate anchors leads to the decrease of the embedment depth loss.
\end{abstract}

\section{Introduction}

Suction embedded plate anchor is a new type of foundation for marine platform structures. It has the advantages of accurate positioning, low cost, easy operation, and high vertical uplift bearing capacity. Thus, it has been extensively used in offshore engineering and has been used as a type of permanent anchor foundation in Angola [1].

When the plate anchor installation is completed by using the suction caisson, the anchor is generally embedded vertically in the seabed, as shown in Figure 1(a) $\left(Z_{\mathrm{i}}\right.$ is the initial embedment depth of the plate anchor, $B$ is the width and $t$ is the thickness of the plate anchor, and $e$ is the anchor padeye eccentricity). Then, the plate anchor is rotated by pulling the mooring line that is attached to the plate anchor (Figures 1(b) and 1(c)), and the plate anchor is finally rotated from vertical to horizontal direction to serve as a foundation (Figure 1(d)). This process is termed "keying" process. During the keying process, the embedment depth of plate anchor will be greatly reduced, which may lead to the decrease of the bearing capacity of anchor. Therefore, the variation of embedment depth and the bearing capacity of plate anchors in the keying process are critical issues in the design of the plate anchor. 


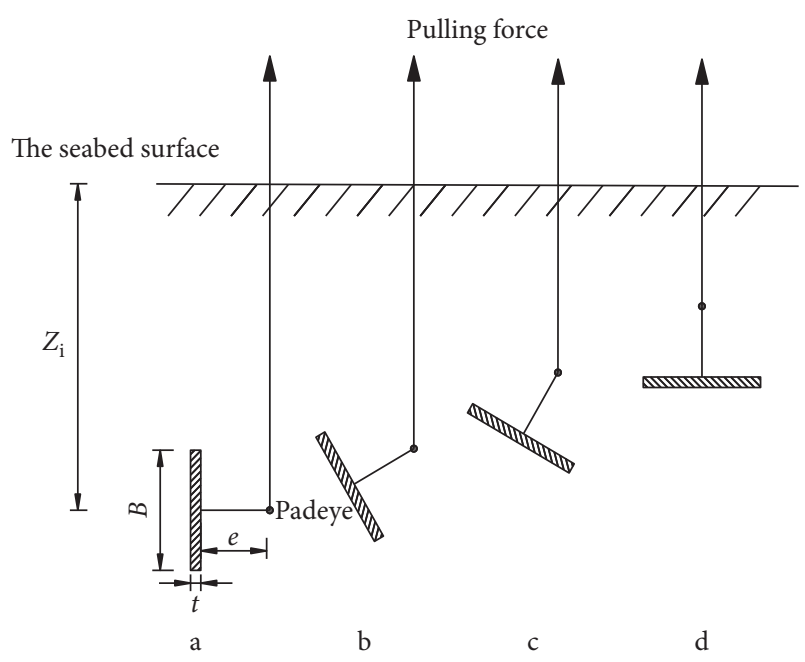

FIgURE 1: Diagram of the keying process of the plate anchor.

The bearing capacity of foundation is an important problem that has been widely studied (e.g., $[2,3])$. Similarly, the pullout capacity of the plate anchors is also a critical problem in ocean engineering. Das [4] and Das and Puri [5] proposed a calculation program to estimate the ultimate pullout capacity of the plate anchors in clay by using the small-scale model test under $1 \mathrm{~g}$ condition. Merifield et al. [6,7] presented rigorous numerical limit analysis for the ultimate capacity of horizontal, vertical, and inclined strip plate anchors in both homogeneous and inhomogeneous clay soils. Similarly, by using the finiteelement limit analysis, the ultimate uplift capacity of multiplate strip anchors and strip anchors embedded adjacent to sloping ground in fully cohesive soil under undrained condition have also been studied [8-11]. These complex interactions between adjacent plates have also been presented in the seismic problems [12]. Zhang et al. [13] investigated the undrained behavior of vertical uplift plate anchors in remolded soft Ariake clay by laboratory tests and finite-element analyses.

Based on the field experiment and laboratory test, Wilde et al. [14] and Wilde [15] derived the load-displacement curve of the keying process of plate anchors and examined the relation between the ultimate pullout capacity of the plate anchor and the soil properties, disturbances, and strain rate. Gaudin et al. [16-18] studied the suction caisson installation, recovery of the suction caisson, and the keying process of plate anchors through a series of centrifuge test. The experimental results were compared with that of the plate anchors directly embedded in the soil before the test, and the effects of the installation of the suction caisson, the recovery of the caisson, and the keying process of plate anchors were studied. O'Loughlin et al. [19] conducted centrifuge tests by using a semimodel to investigate the loss in plate anchor embedment during the keying process. The results show that the keying process will result in the decrease of the embedment depth of plate anchor, and anchor padeye eccentricity has great influence on the displacement of anchor. Song et al. [20, 21] investigated the pullout capacity and rotation behaviour of the plate anchors which were initially installed vertically in homogeneous clay based on model test under 1 and $100 \mathrm{~g}$ conditions and numerical analysis and proposed an influence factor on the embedment loss of the vertical pulling plate anchors. $\mathrm{Yu}$ et al. [22] discussed the influence of anchor padeye eccentricity, pullout angle, and shape of anchor on the embedment loss of plate anchors during keying by numerical simulation. It is concluded that the influence of anchor padeye eccentricity is the greatest and the shape of anchor is the least. Wang et al. [23-25] analyzed the keying process of plate anchors embedded in the normally consolidated clays based on the remeshing and interpolation technique with small strain (RITSS), and they found that the losses of the embedment depth of the plate anchors were mainly dependent on the coupling effect of the anchors roughness coefficient, thickness of plate anchors, and anchor padeye eccentricity. Some researchers (e.g., [26]) also studied the effects of the angle of anchorchain inclination on reducing the embedment depth loss of the plate anchors. Their results showed that with the decrease of the angle of anchor-chain inclination, the depth of the anchors being pulled out decreases linearly. Liu et al. [27] proposed a plasticity model of three-dimensional plate anchors with consideration of shank and flap. The effects of the flap on the embedment loss and pullout capacity, and the effects of loading eccentricity and pullout angle on diving behavior were investigated. Based on discrete-element method (DEM) simulations, Evans and Zhang [28] investigated the behavior of plate anchors during pullout from the view of microscale physical processes. The observations of the simulated contact force network and particle velocity during uplift were used to provide insight into anchor failure mechanisms. In addition, Singh and Ramaswamy $[29,30]$ performed model test of plate anchors under cyclic loadings and investigated the effects of the dynamic loading level, the frequency of cyclic loading, and the preloading level on the embedment depth loss. Rahimi et al. [31] performed a series of near-full-scale experiments and numerical models on plate anchors embedded in sand with and without geocell reinforcement, and the influence of geocell reinforcement on the uplift capacity of plate anchors was examined.

In summary, current studies on the keying process of plate anchors mainly focused on the homogeneous soil or the normally consolidated soil. However, the deep-sea soil layers are quite complex. In emerging provinces and fields, highly layered soils are prevalent and quite a number of stratified seabed profiles involve interbedded layers displaying strong variations in shear strength [32]. Therefore, this study utilized the three-dimensional Coupled Eulerian Lagrangian (CEL) large deformation finite element analysis to investigate the keying process of plate anchors on the more complicated layered soil, i.e., the three-layer soft-stiffsoft clay deposits. The flow of soil and the embedment depth loss of plate anchors in the keying process were analyzed, and the effects of soil strengths of different layers were investigated. 


\section{Validation of the Numerical Model}

To validate the reliability of CEL method in predicting the interaction between plate anchors and soil, the loss of the embedment depth during keying process obtained by numerical calculation was compared with that from the existing centrifuge model test [20]. The numerical analyses were performed using the FE package ABAQUS/Explicit [33]. The feature of fixed Eulerian mesh and the contact between the Eulerian and Lagrangian materials allows for effective application of the CEL approach in large deformation geotechnical problems. The soil was assumed to be completely saturated and elastoplastic, and the MohrCoulomb model was adopted.

The centrifuge tests [20] were carried out by using the drum centrifuge which has a diameter of $1.2 \mathrm{~m}$, a channel of radial depth of $0.2 \mathrm{~m}$, and a height of $0.3 \mathrm{~m}$ to study the relation between the anchor padeye eccentricity of pulling force and the loss of the embedment depth. A uniform clay soil deposit was considered with an undrained shear strength, $S_{\mathrm{u}}=18 \mathrm{kPa}$, and a submerged unit weight of $9.5 \mathrm{kN} / \mathrm{m}^{3}$. The stainless-steel square plate anchor in the model test has a thickness of $2 \mathrm{~mm}$, a length of $40 \mathrm{~mm}$, and an eccentricity of $25 \mathrm{~mm}$. The test was conducted at $100 \mathrm{~g}$ to model a prototype plate anchor of $4 \mathrm{~m} \times 4 \mathrm{~m} \times 0.2 \mathrm{~m}$, and an eccentricity of $2.5 \mathrm{~m}$. The submerged unit weight of the anchor is $17.0 \mathrm{kN} / \mathrm{m}^{3}$. The anchor was installed into the soil vertically and was pulled out vertically. The normalized initial embedment of the anchor is $Z_{\mathrm{i}} / B=3$.

Following the previous studies, the loss of the embedment in this paper refers to the centroid displacement of plate anchor $(\Delta Z)$. The normalized pulling force is defined as $F /$ $S_{\mathrm{u}} A$, where $F$ is the vertical pulling force on the plate anchor, and $A$ is the plate area. Figure 2(a) shows the variation of normalized pulling force with the normalized displacement of the anchor padeye $(d / B)$ in this study. Also shown in the figure are the results from the model test [20] and the RITSS method [23]. It can be seen that the normalized pulling forces obtained from CEL technique are between the results of RITSS method and the results of model test. Figure 2(b) presents the variation of the inclination of the plate anchor $(\beta)$ with the normalized loss of the embedment during keying process of plate anchors $(\Delta Z / B)$. The loss of the embedment depth of plate anchors from two types of numerical simulation is larger than the results from model tests. This may be related to the neglection in the numerical model of both the anchor shank and the embedded disturbances to soil of the plate anchor in the model test. In addition, the model anchor reached its stable state at an inclination of $10^{\circ}$ from the horizontal direction. If the final orientation angle of the anchor is also taken as $10^{\circ}$ in the numerical analyses, the loss of embedment would be much similar to the model test.

The second validation is for the model test results from O'Loughlin et al. [19], in which the keying process of the plate anchors in a normally consolidated clay was modeled by using the drum centrifuge. The undrained shear strength of the soil increases linearly with depth. The shear strength of soil on the surface of seabed is zero, and the rate of increase of shear strength is $0.7 \mathrm{kPa} / \mathrm{m}$. The submerged unit weight of

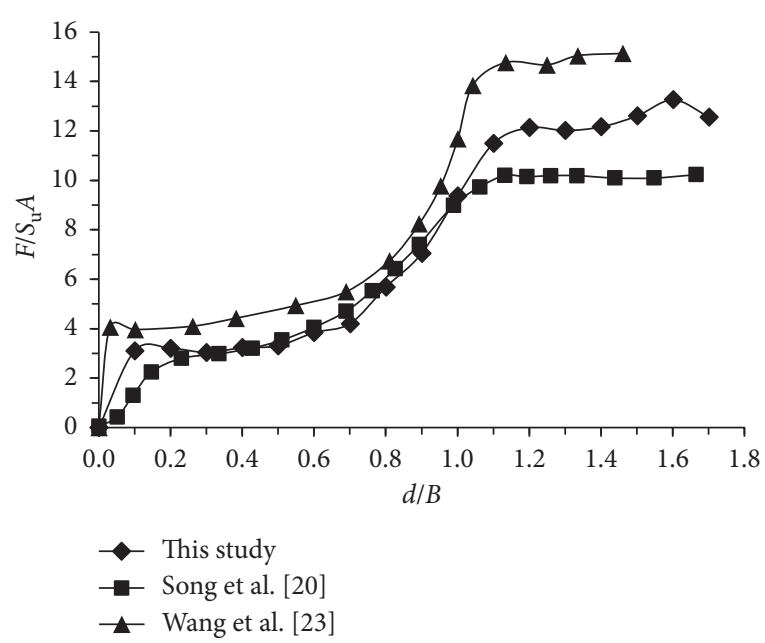

(a)

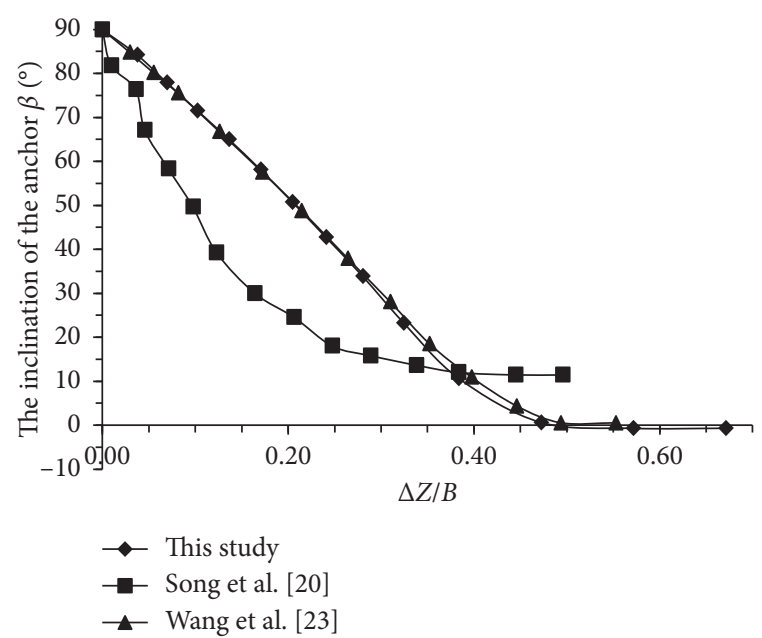

(b)

Figure 2: Comparison with test results by Song et al. [20] and with the numerical results by Wang et al. [23] for uniform soil: (a) normalized load-displacement relationship; (b) variations of plate inclinations with the normalized embedment loss.

soil and plate anchor is 6.5 and $67 \mathrm{kN} / \mathrm{m}^{3}$, respectively. The width of the plate anchor is $B=3 \mathrm{~m}$, the ratio of thickness to width is $t / B=0.067$, and the eccentricity ratio of plate anchor $e / B$ is 0.50 . The normalized initial embedment of the anchor is $Z_{\mathrm{i}} / B=3$. The loss of the embedment depth of plate anchors from CEL method and model test is shown in Figure 3. It can be seen that the numerical results in this study are generally close to the test, except for the stages when the keying process is nearly finished. Similar to the results of uniform soil, this is due to that the model anchor in the test reached its stable state with an inclination and not in the horizontal direction, resulting in some differences in the embedment loss compared to the numerical results.

\section{Model Parameters}

The soil in the model consists of three layers, i.e., the top and bottom layers of normally consolidated soil and the interbedded stiff layer of uniform soil (Figure 4). The 


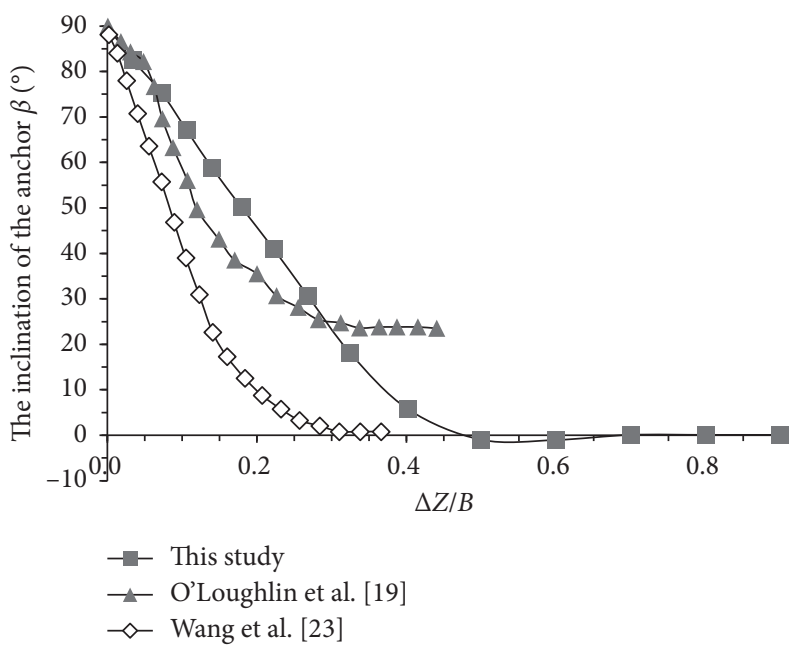

Figure 3: Comparison with test results by O’Loughlin et al. [19] and with the numerical results by Wang et al. [23] for normally consolidated soil.

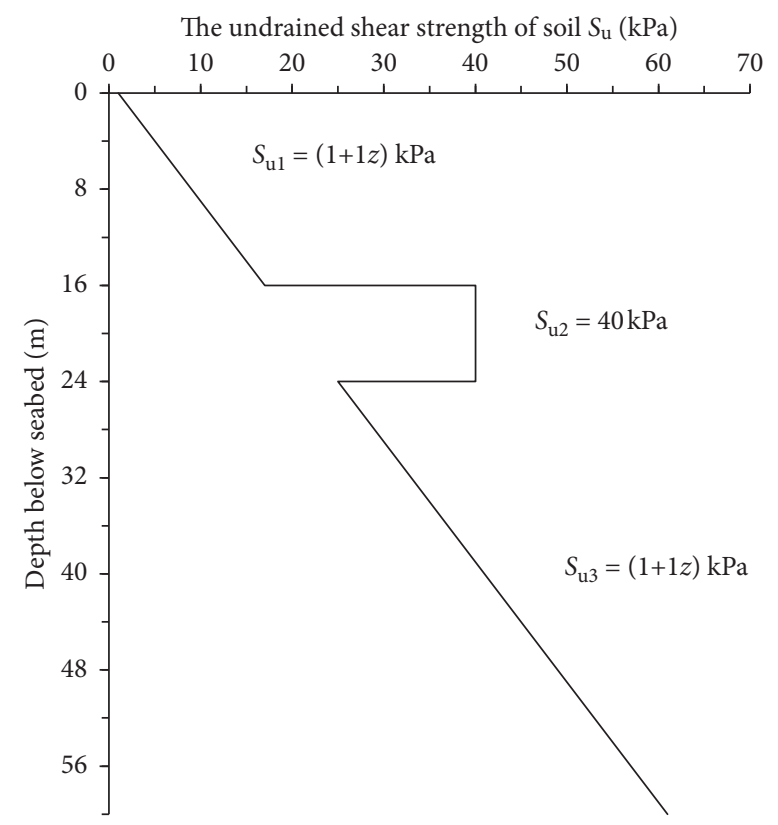

FIGURE 4: Typical seabed profiles with normally consolidated clay and an interbedded stiff layer.

undrained shear strength of the top and bottom soil layers increases linearly with depth as $S_{\mathrm{u}}=(1+k z) \mathrm{kPa}$, where $k$ is the rate of increase of shear strength $(\mathrm{kPa} / \mathrm{m})$ and $z$ is the depth $(\mathrm{m})$. The undrained shear strength of the uniform stiff soil is $S_{\mathrm{u}}=40 \mathrm{kPa}$. The effective unit weight for soils is $6 \mathrm{kN} / \mathrm{m}^{3}$. Young's modulus $(E)$ is taken as $E / S_{\mathrm{u}}=500$ and a Poisson's ratio of 0.49 is adopted to give minimal volumetric strains, while maintaining numerical stability [32]. The plate anchor is a rigid body, and the submerged unit weight is $67.0 \mathrm{kN} / \mathrm{m}^{3}$.

The three-dimensional (3D) elements were used in the CEL analysis. For the parametric studies, only a half of the domain was involved in the analyses due to the symmetry inherent. The roughness factor of soil-anchor interface was taken as 0.3 . The soil and plate anchor were discretized using Eulerian hexahedral elements of type EC3D8R and continuum elements of types C3D8R (hexahedron), respectively. The soil layers underlay an initially void layer to accommodate the possible soil heave resulting from keying process of the plate anchor. This void layer has no weight and strength, but it can provide an additional domain to accommodate the soil flow during the large deformations. The length, width, and thickness of the plate anchor are $L=4 \mathrm{~m}, B=4 \mathrm{~m}$, and $t=0.2 \mathrm{~m}$, respectively. The numerical model of the plate anchor and soil profiles is shown in Figure 5. The soil domain is chosen as $10 B$ in length and $5 B$ in width, which is shown to be sufficiently large to avoid boundary effect. 


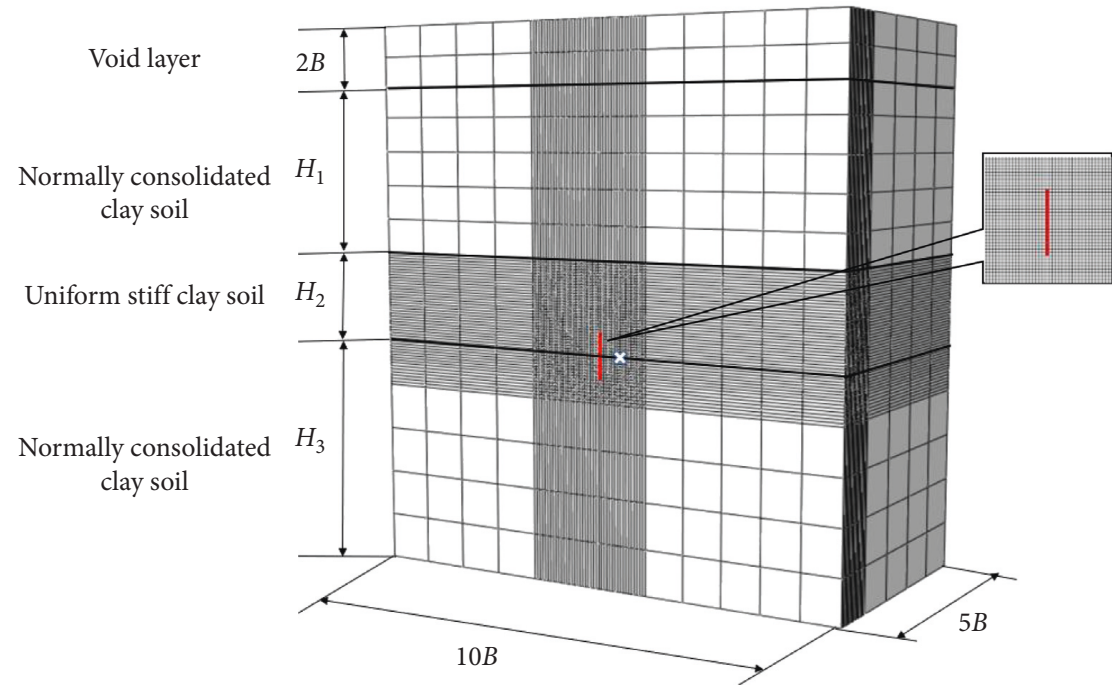

FIgURE 5: Mesh of the numerical model.

Similar domain sizes were considered by Zheng et al. [34] in spudcan continuous penetration analysis. For the selection of element size, routine preliminary simulations are carried out, varying the minimum element size around the plate anchor, $h_{\min } / B$. Balancing the computational effort and accuracy of the computed results, and also complying with existing studies (e.g., Zheng et al. [34]), $h_{\min } / B=0.025$ is adopted.

\section{Soil Flow Mechanism}

The keying process of the plate anchors is a process combining translation and rotation and involves problems of highly nonlinear and large deformation. The soil generally flows faster around plate anchors during keying rotation process and the flow of soil has great influence on the loss of the embedment depth and bearing capacity of the plate anchors. In this section, the soil flow mechanism of plate anchors with various initial embedment depths in normally consolidated soil and three-layer soft-stiff-soft clay deposits was studied.

Figure 6 shows the soil flow around plate anchors in normally consolidated and layered soil for an initial embedment depth of $Z_{\mathrm{i}}=3.5 \mathrm{~B}$ (i.e., $14 \mathrm{~m}$ ). The plate anchor is thus located at the bottom of first soil layer and is close to the middle stiff soil layer for the three-layer deposits. It can be found that the flow of soil around the rotating plate anchor in the three-layer deposits is faster than the normally consolidated soil as a result of the presence of the underlying stiff layer. The backflow soils strike the plate of anchors, and a large velocity difference is formed on the upper and lower faces of the plate anchor. This accelerates the keying process of the plate anchors, which is conducive to reduce the loss of the embedment depth.

Figure 7 shows the soil flow around plate anchors in normally consolidated and layered soil for the initial embedment depth of $Z_{\mathrm{i}}=7.0 \mathrm{~B}$ (i.e., $28 \mathrm{~m}$ ) when the keying process is completed (i.e., the plate anchor is horizontal). In this case, the plate anchor is buried in the third soil layer ( $4 \mathrm{~m}$ away from the soil interface), the upward flow for layered soil is slower than that in the normally consolidated soil, which slightly reduces the loss of the embedment depth of plate anchors.

\section{Parametric Study}

5.1. Effect of the Thickness of Top Soil Layer. The influence of soil thickness of the top soil layer on the rotation characteristics of the plate anchor was analyzed. The thickness of the top soil layer $H_{1}$ was selected as $8 \mathrm{~m}, 12 \mathrm{~m}, 16 \mathrm{~m}, 20 \mathrm{~m}$, and $24 \mathrm{~m}$, respectively. Considering that the thickness of the top soil layer may have different effects on plate anchors at different initial embedded depths, three typical initial embedment positions were selected, as shown in Figure 8 . Among them, $P_{1}$ is located at the interface of first and second soil layers, $P_{2}$ is located at the middle of second soil layer, and $P_{3}$ is located at the interface of second and third soil layers. In order to study the rotation characteristics of plate anchors at different positions, the variations of the inclination of the anchor plate with the normalized loss of the embedment depth $(\Delta Z / B)$ are examined.

Figure 9(a) shows the variation of plate inclinations with the loss of the embedment depth of plate anchors at position $P_{2}$ for different thicknesses of the first soil layer $\left(H_{1}\right)$, and Figure 9(b) shows the ultimate loss of the embedment depth at the end of keying process of plate anchor $\left(\Delta Z_{\mathrm{u}}\right)$. We can see the loss of the embedment depth decreases with the increase of the thickness of the first soil layer. The maximum embedment loss is about $1.10 \mathrm{~B}$ and $0.75 \mathrm{~B}$ for the thickness of the first soil layer of $H_{1}=8 \mathrm{~m}$ and $H_{1}=24 \mathrm{~m}$, respectively. When the thickness of the first soil layer increases to $16 \mathrm{~m}$, its influence on the rotation characteristics of the plate anchor is limited.

Figure 10 shows the variation of plate inclinations with the loss of the embedment depth of plate anchors at positions $P_{1}$ and $P_{3}$ during keying process for different $H_{1}$ values. 


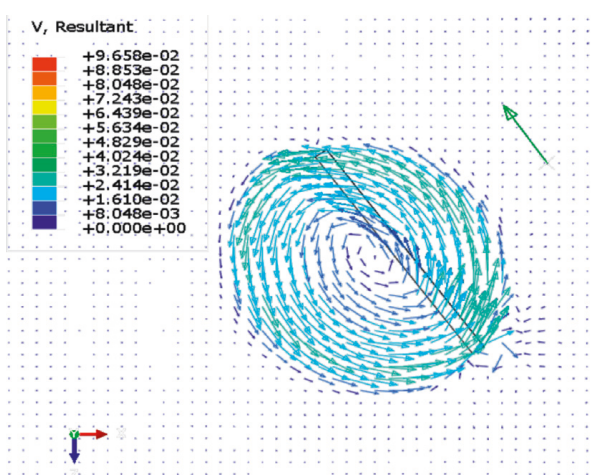

(a)

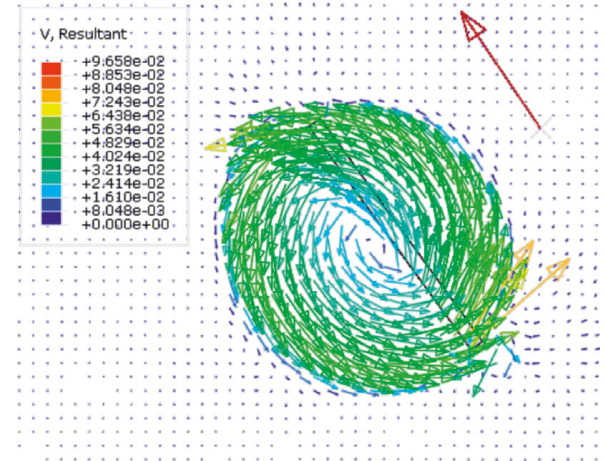

(b)

FiguRE 6: The soil flow around plate anchors in (a) normally consolidated soil $\left(Z_{\mathrm{i}}=14 \mathrm{~m} ; \beta \approx 55^{\circ}\right)$ and (b) layered soil $\left(Z_{\mathrm{i}}=14 \mathrm{~m} ; \beta \approx 55^{\circ}\right)$.

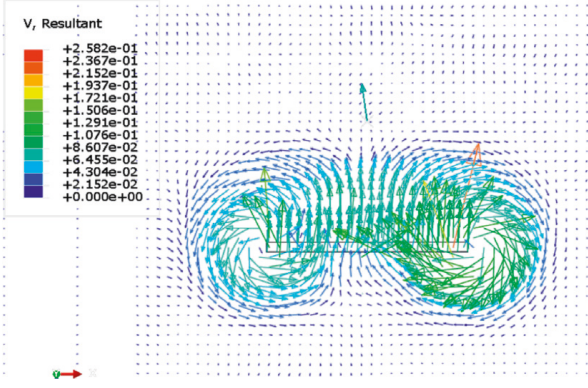

(a)

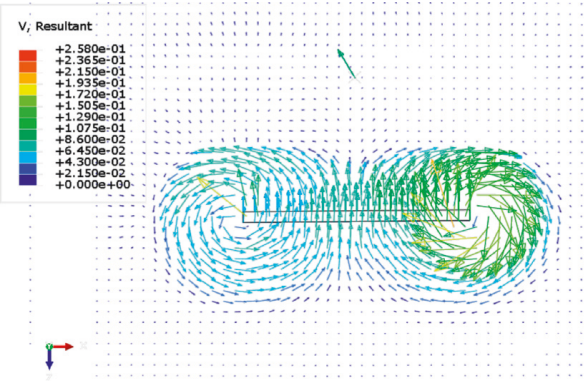

(b)

Figure 7: The soil flow around plate anchors in (a) normally consolidated soil $\left(Z_{\mathrm{i}}=28 \mathrm{~m}\right)$ and (b) layered soils $\left(Z_{\mathrm{i}}=28 \mathrm{~m}\right)$ when the keying process is completed.

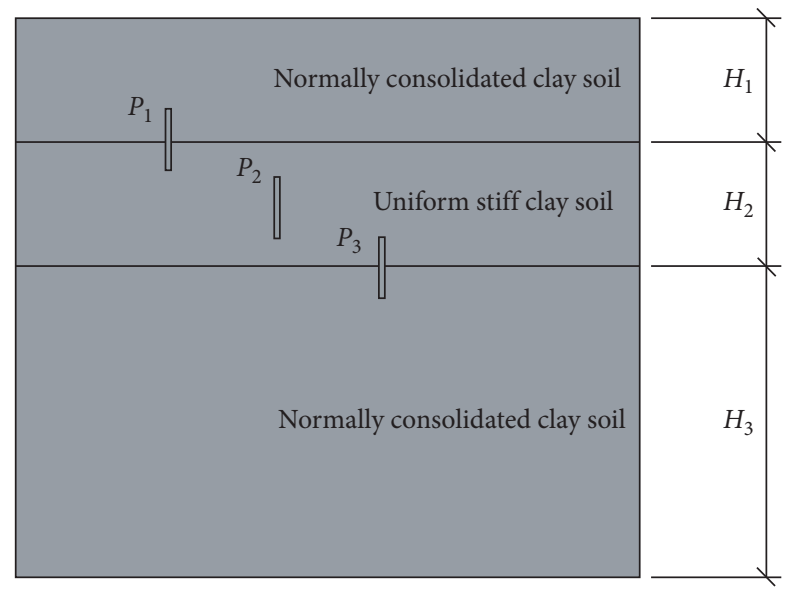

FIGURE 8: Initial embedded positions of plate anchors.

Observe that the effect of the thickness of the first soil layer on the rotation process of plate anchor is negligible when the plate anchor is initially buried at the interface of first and second soil layers or at the interface of second and third soil layers. In addition, the ultimate loss of the embedment depth is similar for the two positions (about $0.60 \mathrm{~B}$ for the plate anchor at position $P_{1}$ and $0.70 B$ for the plate anchor at position $P_{3}$ ).
5.2. Effect of Initial Embedment Depth. Figure 11 shows the variation of plate inclinations with the loss of the embedment depth for plate anchors initially buried in the first, second, and third soil layers, respectively. Observe that when the plate anchor is initially buried in the first soil layer, the embedment loss of the plate anchor increases with the increase of the initial embedment depth $Z_{\mathrm{i}}$. The embedment loss is minimum for $Z_{\mathrm{i}}=8 \mathrm{~m}$ (about $0.37 B$ ), and the 


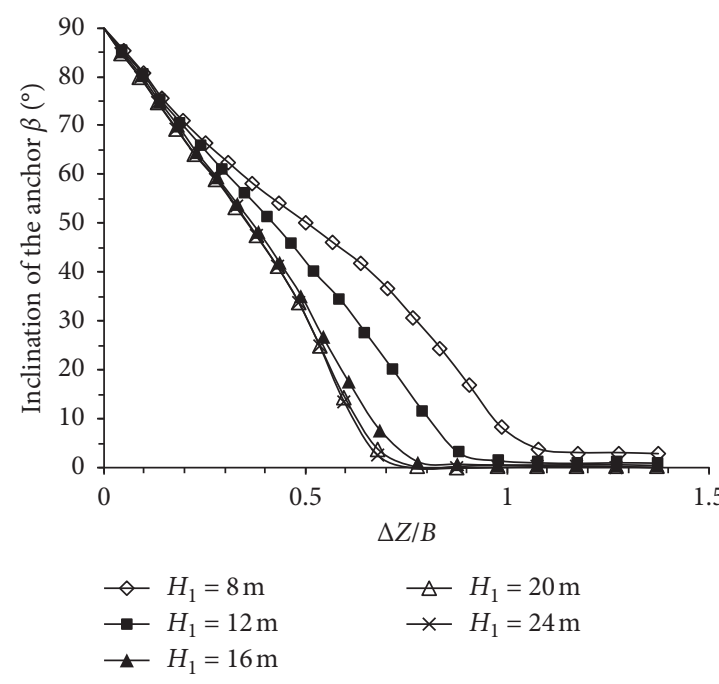

(a)

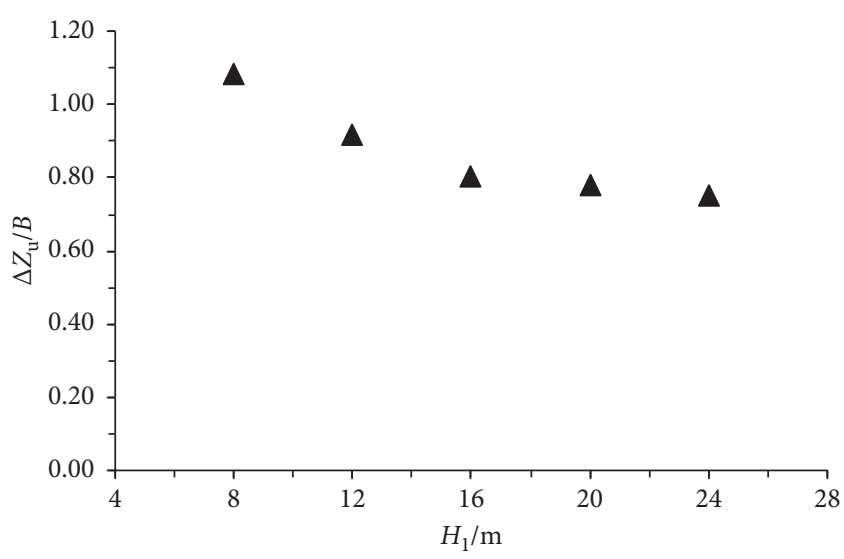

(b)

FIGURE 9: Influence of top soil layer thickness on the plate anchors at position $P_{2}$ : (a) variations of plate inclinations with the embedment loss; (b) the embedment loss for various $H_{1}$ values.

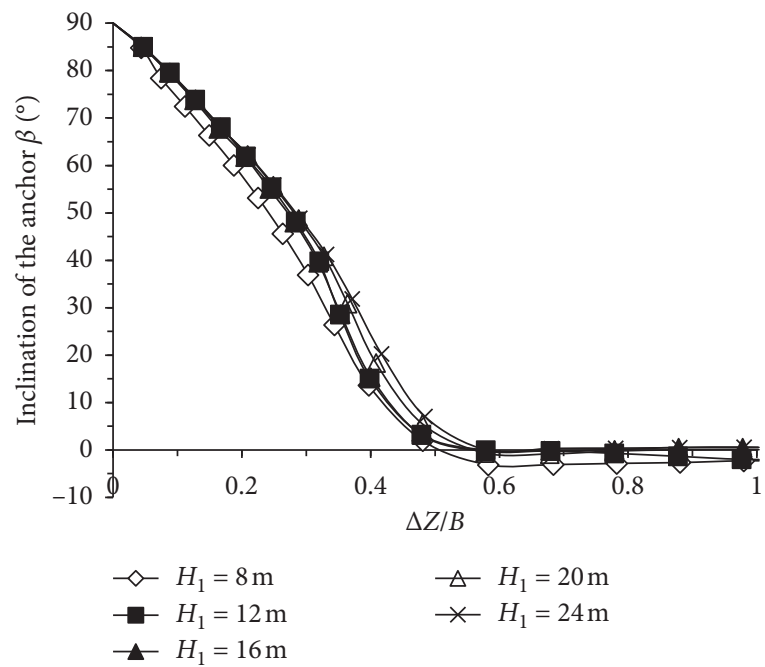

(a)

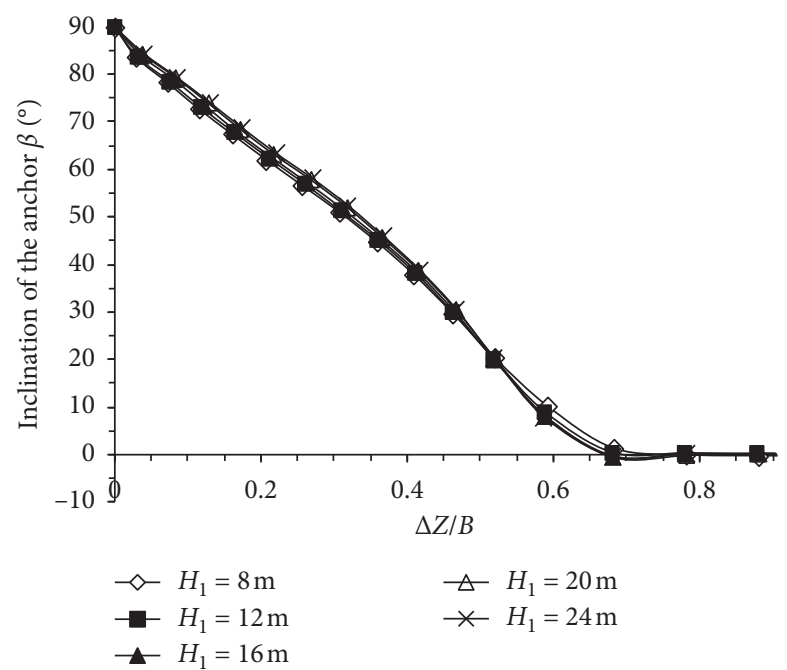

(b)

Figure 10: Influence of top soil layer thickness on the plate anchors at position $P_{1}$ and $P_{3}$. (a) The rotation of position $P_{1}$; (b) the rotation of position $P_{3}$.

maximum embedment loss is about $0.57 \mathrm{~B}$ for $Z_{\mathrm{i}}=16 \mathrm{~m}$. When the plate anchor is buried in the second soil layer, the embedment loss increases initially and then decreases with the increase of the initial embedment depth of plate anchors. When the plate anchor is located in the third soil layer, the influence of the initial embedment depth is negligible, with the maximum embedment loss of the plate anchor of about $0.7 B$.

Figure 12(a) shows the ultimate loss of embedment depth of plate anchors at different initial embedment depths in layered and normally consolidated soils. It can be seen that the influence of the interbedded uniform stiff soil layer on the embedment depth loss is significant for plate anchors embedded at $12 \mathrm{~m} \sim 32 \mathrm{~m}$ (i.e., from $1.0 B$ above to $2.0 \mathrm{~B}$ below the stiff soil layer). When the plate anchor is initially buried at other depths, the influence of the interbedded stiff soil layer is almost negligible. The embedment depth loss of plate anchors in the three-layer soft-stiff-soft and normally consolidated soil can be normalized as

$$
\gamma_{Z}=\frac{\Delta Z_{\mathrm{u}}}{\Delta Z_{\mathrm{u}}^{\prime}}
$$

where $\Delta Z_{\mathrm{u}}$ and $\Delta Z_{\mathrm{u}}^{\prime}$ are the ultimate embedment depth loss of plate anchors in layered soil and normally consolidated soil, respectively. The influence of the interbedded stiff soil 


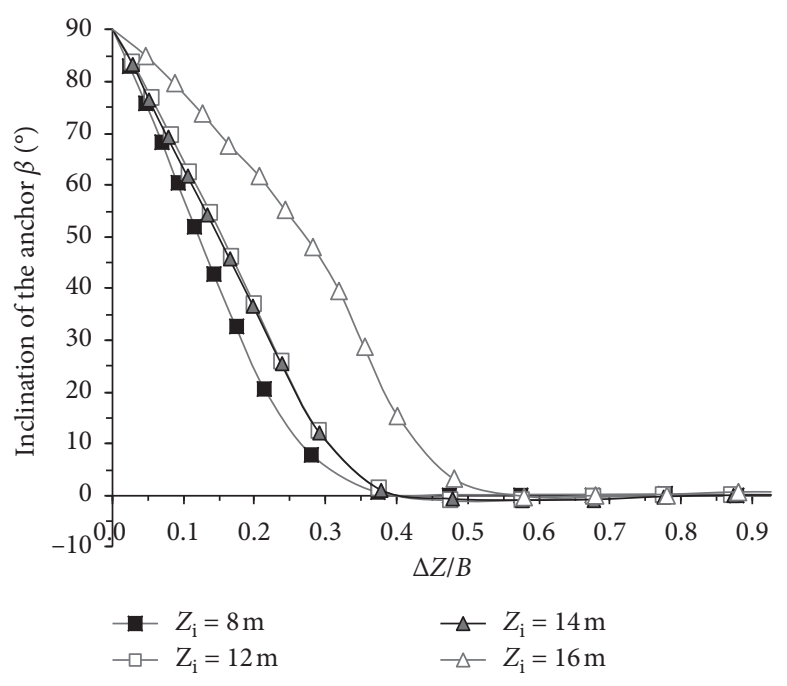

(a)

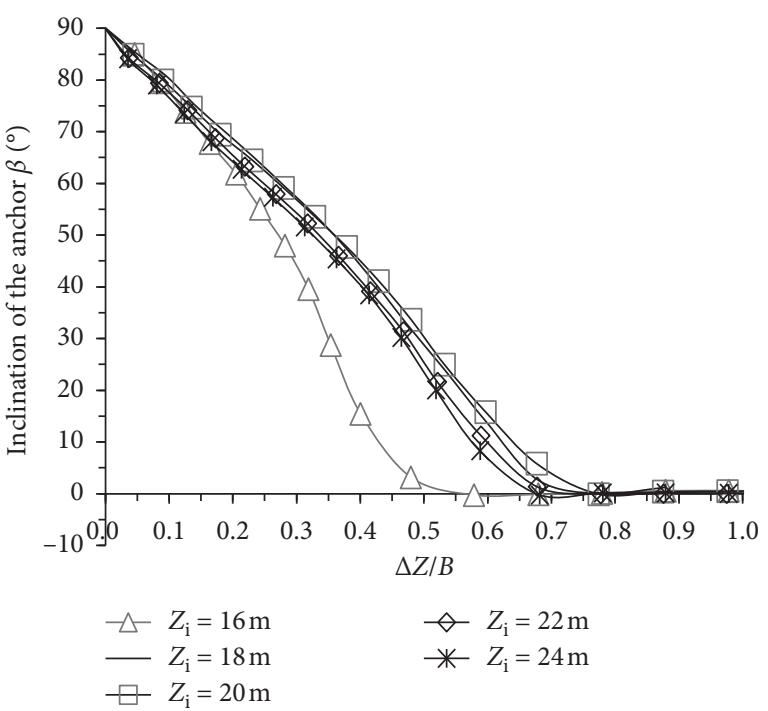

(b)

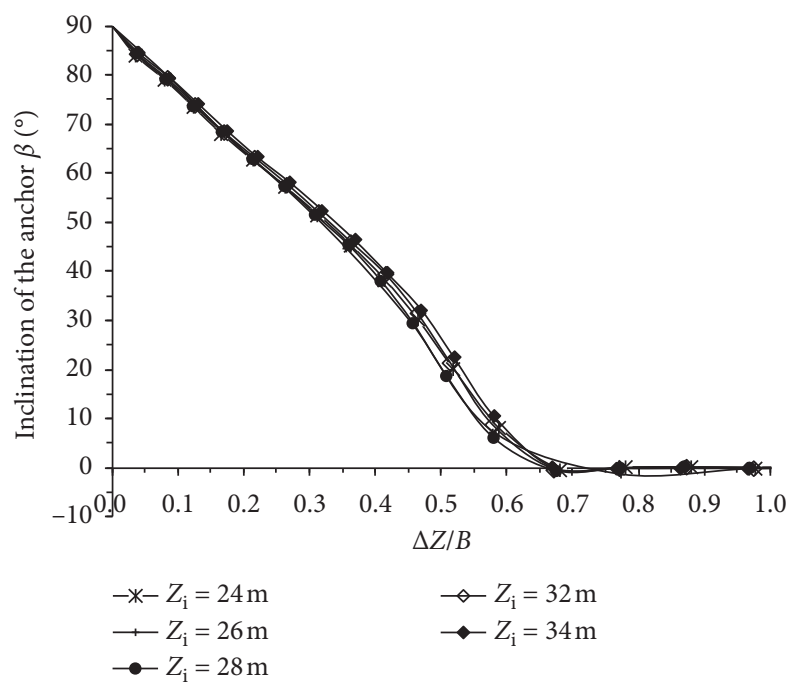

(c)

Figure 11: Variations of plate inclinations with the embedment loss in different soil layers. Plate anchor initially buried in (a) the top soil layer, (b) the middle soil layer, and (c) the bottom soil layer.

layer on the embedment loss of plate anchor is further shown in Figure 12(b). This result is useful for the further estimation on the variation of the uplift bearing capacity of plate anchors.

5.3. Effect of the Shear Strength of Layered Soil. The keying process of plate anchors initially embedded in the middle of interbedded uniform stiff soil layer was investigated for soil layers with different shear strengths. The first case is for different undrained shear strengths of the stiff layer $S_{\mathrm{u} 2}=30$, $35,40,45$, and $50 \mathrm{kPa}$ and the strength gradient of the normally consolidated layers of $1.0 \mathrm{kPa} / \mathrm{m}$. The other case is for $S_{\mathrm{u} 2}=40 \mathrm{kPa}$ and the strength gradient of the normally consolidated layers of $k=0.5,0.8,1.0,1.5$ and $2.0 \mathrm{kPa} / \mathrm{m}$, respectively. The results for different cases are shown in Figure 13.
For the first case (Figure 13(a)), the embedment depth loss of plate anchors increases significantly with the increase of the $S_{\mathrm{u} 2}$. The minimum ultimate embedment depth loss is about $0.70 \mathrm{~B}$ for $S_{\mathrm{u} 2}=30 \mathrm{kPa}$, and the maximum ultimate embedment depth loss is about $1.0 B$ for $S_{\mathrm{u} 2}=50 \mathrm{kPa}$. For the second case (Figure 13(b)), the embedment depth loss of plate anchors decreases with the increase of the strength gradient $(k)$ of the normally consolidated layer, and it is essentially in the range of $0.7 \mathrm{~B}$ and $0.9 \mathrm{~B}$. Note that this is different from the observation of Wang et al. [23], in which they found that the embedment depth loss increases with the increase of $k$ in normally consolidated soil. Therefore, it is inferred that the increase of the strength of local soil around the plate anchors will lead to the increase of the embedment depth loss, but the increase of the strength of soil slightly away from the plate anchors may lead to the decrease of the embedment depth loss. 


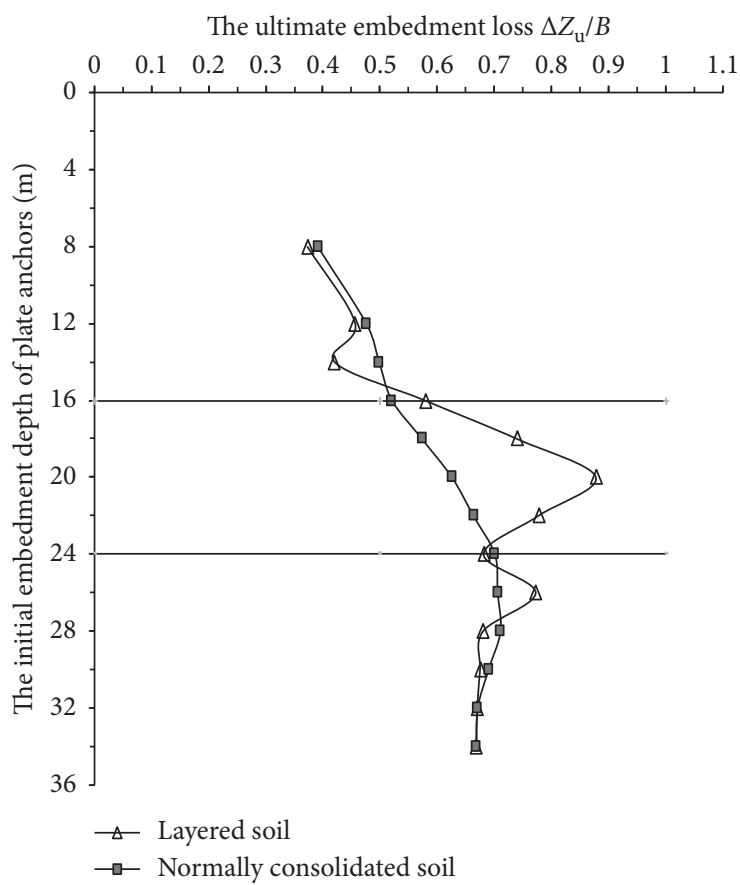

(a)

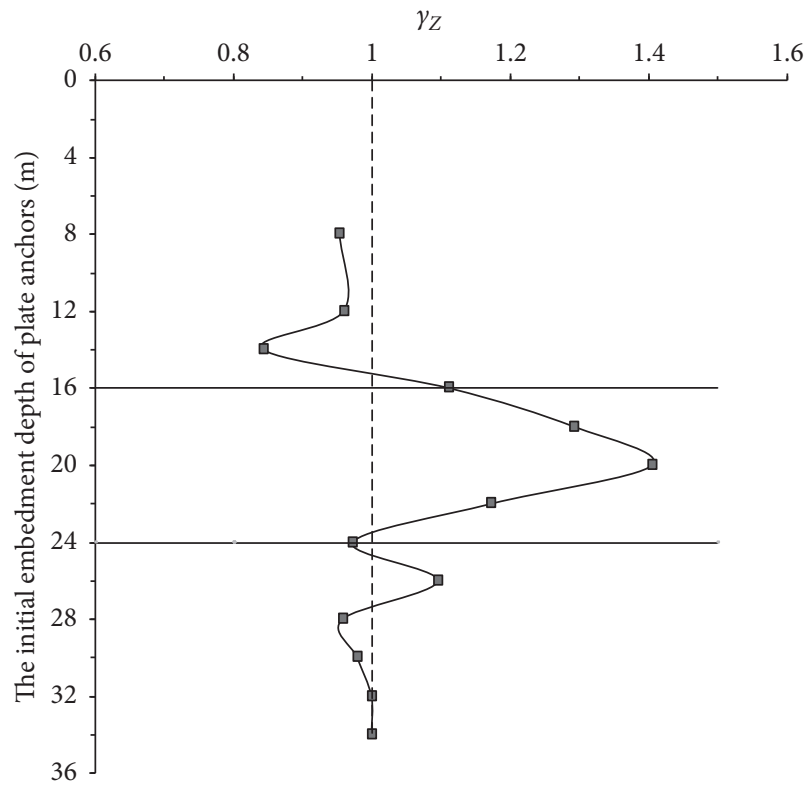

(b)

FIGURE 12: Comparison of the ultimate embedment loss in layered soil and normal consolidated soil: (a) effect of initial embedment depth on the ultimate embedment loss; (b) effect of layered soil on the embedment.

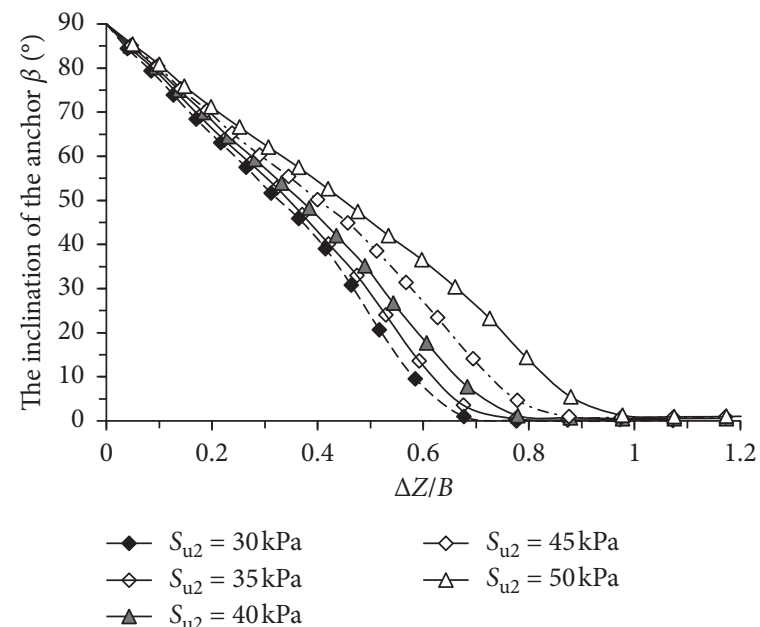

(a)

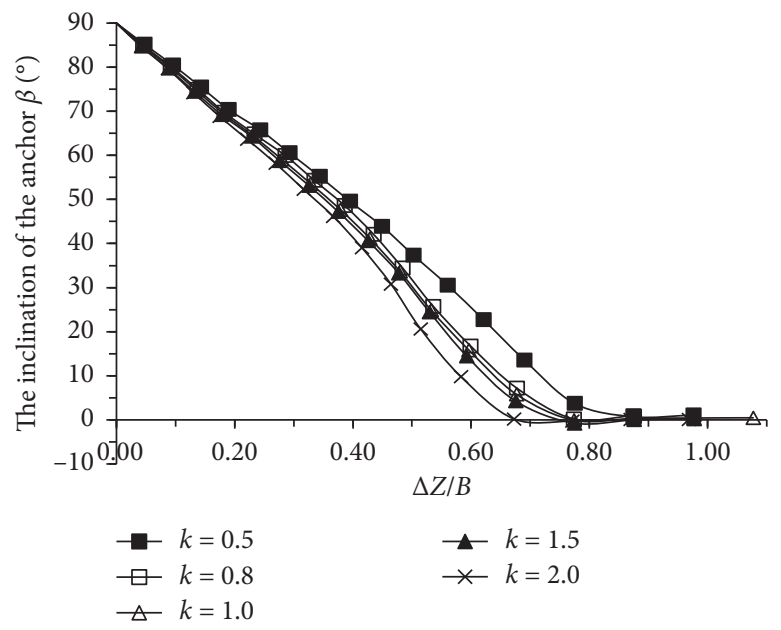

(b)

FIGURE 13: The effect of soil strength on the keying process of plate anchors: (a) effect of the soil strength of middle stiff soil layer; (b) effect of the strength gradient of top soil layers.

\section{Conclusions}

In this study, the keying process of plate anchors in threelayer soft-stiff-soft clay deposits was investigated by using the CEL approach to perform the large deformation numerical simulation. The soil flow mechanism around the plate anchors and the influence of a series of parameters of layered soil on the embedment depth loss were examined. The results were compared with those from the traditional results of normally consolidated soil. The key conclusions are as follows:

(1) The influence of the thickness of the top soil layer is significant on the keying process of plate anchors buried at the middle of the interbedded stiff soil layer. With the increase of the thickness of the top soil layer, the loss of embedment depth of plate anchors decreases. The influence of the thickness of 
the top soil layer on the rotation process of plate anchor is negligible when the plate anchor is initially buried at the interface of top and stiff soil layers or at the interface of stiff and bottom soil layers.

(2) When the plate anchors are buried at the top soil layer and near the stiff soil layer, the surrounding soil flow velocity is larger and the embedment depth loss of plate anchors is smaller. Compared with the anchors buried in the case of normally consolidated soils, the interbedded stiff soil layer affects the keying process of plate anchors embedded in $1.0 B$ above and $2.0 B$ below the interbedded stiff soil layer.

(3) The strength gradient of the normally consolidated soil layers and strength of the uniform stiff soil layer have significant effects on the keying process of plate anchors. With the increase of local soil strength around the plate anchor, the loss of embedment depth increases, but the loss of embedment depth decreases with the increase of strength gradient of the normally consolidated soils, which is different from previous observations on the normally consolidated soils.

\section{Data Availability}

The data used to support the findings of this study are available from the corresponding author upon request.

\section{Conflicts of Interest}

The authors declare that they have no conflicts of interest.

\section{Acknowledgments}

This research has been supported by the Fundamental Research Funding Scheme for the Central Universities in China (grant nos. 2018B42414, 2016B01114, and 2018B57014), the National Natural Science Foundation of China (grant nos. 41602280, 51808193, and 51778211), Jiangsu Province Postdoctoral Science Foundation (grant no. 2018K044B), and China Postdoctoral Science Foundation (grant no. 2016M601708). These supports are gratefully acknowledged.

\section{References}

[1] R. P. Brown, P. C. Wong, and J. M. Audibert, "SEPLA keying prediction method based on full-scale offshore tests," in Proceedings of 2nd International Symposium on Frontiers in Offshore Geotechnics, pp. 717-722, Perth, Australia, November 2010.

[2] Y. Wu, X. Zhou, Y. Gao, L. Zhang, and J. Yang, "Effect of soil variability on bearing capacity accounting for non-stationary characteristics of undrained shear strength," Computers and Geotechnics, vol. 110, pp. 199-210, 2019.

[3] J. Zhang, Y. Gao, T. Feng, J. Yang, and F. Yang, "Upper-bound finite-element analysis of axisymmetric problems using a mesh adaptive strategy," Computers and Geotechnics, vol. 102, pp. 148-154, 2018.

[4] B. M. Das, "A procedure for estimation of ultimate uplift capacity of foundations in clay," Soils and Foundations, vol. 20, no. 1, pp. 77-82, 1980.
[5] B. M. Das and V. K. Puri, "Holding capacity of inclined square plate anchors in clay," Soils and Foundations, vol. 29, no. 3, pp. 138-144, 1989.

[6] R. S. Merifield, S. W. Sloan, and H. S. Yu, "Stability of plate anchors in undrained clay," Géotechnique, vol. 51, no. 2, pp. 141-153, 2001.

[7] R. S. Merifield, A. V. Lyamin, and S. W. Sloan, "Stability of inclined strip anchors in purely cohesive soil," Journal of Geotechnical and Geoenvironmental Engineering, vol. 131, no. 6, pp. 792-799, 2005.

[8] J. Kumar and J. P. Sahoo, "Vertical uplift resistance of a group of two coaxial anchors in clay," Journal of Geotechnical and Geoenvironmental Engineering, vol. 138, no. 3, pp. 419-422, 2012.

[9] J. P. Sahoo and J. Kumar, "Vertical uplift resistance of two interfering horizontal anchors in clay," Journal of Geotechnical and Geoenvironmental Engineering, vol. 140, no. 4, article 06013007, 2014.

[10] R. S. Merifield and C. C. Smith, "The ultimate uplift capacity of multi-plate strip anchors in undrained clay," Computers and Geotechnics, vol. 37, no. 4, pp. 504-514, 2010.

[11] J. P. Sahoo and S. Khuntia, "Lower bound solutions for uplift capacity of strip anchors adjacent to sloping ground in clay," Marine Georesources \& Geotechnology, vol. 36, no. 4, pp. 405-416, 2017.

[12] J. Song, Q. Fan, T. Feng, Z. Chen, J. Chen, and Y. Gao, “A multi-block sliding approach to calculate the permanent seismic displacement of slopes," Engineering Geology, vol. 255, pp. 48-58, 2019.

[13] N. Zhang, H.-N. Wu, J. S.-L. Shen, T. Hino, and Z.-Y. Yin, "Evaluation of the uplift behavior of plate anchor in structured marine clay," Marine Georesources \& Geotechnology, vol. 35, no. 6, pp. 758-768, 2017.

[14] B. Wilde, H. Treu, and T. Fulton, "Field testing of suction embedded plate anchors," in Proceedings of 11th International Offshore and Polar Engineering Conference, pp. 544-551, Stavanger, Norway, June 2001.

[15] B. Wilde, "Program of centrifuge and field tests on the suction embedded plate anchor," Rep. to SEPLA Joint Industry Project, InterMoor, Houston, TX, USA, 2005.

[16] C. Gaudin, C. D. O’Loughlin, M. F. Randolph, and A. C. Lowmass, "Influence of the installation process on the performance of suction embedded plate anchors," Géotechnique, vol. 56, no. 6, pp. 381-391, 2006.

[17] C. Gaudin, K. H. Tham, and S. Ouahsine, "Plate anchor failure mechanism during keying process," in Proceedings of 18th International Offshore and Polar Engineering Conference, pp. 613-619, Vancouver, Canada, July 2008.

[18] C. Gaudin, M. Simkin, D. J. White, and C. D. O’Loughlin, "Experimental investigation into the influence of a keying flap on the keying behaviour of plate anchors," in Proceedings of 20th International Offshore and Polar Engineering Conference, pp. 533-540, Beijing, China, June 2010.

[19] C. D. O'Loughlin, A. C. Lowmass, C. Gaudin, and M. F. Randolph, "Physical modelling to assess keying characteristics of plate anchors," in Proceedings of the Sixth International Conference on Physical Modelling in Geotechnics, pp. 659-665, Hong Kong, China, August 2006.

[20] Z. Song, Y. Hu, D. Wang, and C. D. O'Loughlin, "Pullout capacity and rotational behaviour of square anchors," in International Conference on Physical Modelling in Geomechanics, pp. 1325-1331, Hong Kong, China, January 2006.

[21] Z. Song, Y. Hu, C. O’Loughlin, and M. F. Randolph, "Loss in anchor embedment during plate anchor keying in clay," 
Journal of Geotechnical and Geoenvironmental Engineering, vol. 135, no. 10, pp. 1475-1485, 2009.

[22] L. Yu, J. Liu, X.-J. Kong, and Y. Hu, "Three-dimensional numerical analysis of the keying of vertically installed plate anchors in clay," Computers and Geotechnics, vol. 36, no. 4, pp. 558-567, 2009.

[23] D. Wang, Y. Hu, and M. F. Randolph, "Keying of rectangular plate anchors in normally consolidated clays," Journal of Geotechnical and Geoenvironmental Engineering, vol. 137, no. 12, pp. 1244-1253, 2011.

[24] D. Wang, C. Gaudin, and M. F. Randolph, "Large deformation finite element analysis investigating the performance of anchor keying flap," Ocean Engineering, vol. 59, no. 2, pp. 107-116, 2013.

[25] D. Wang, Y. Hu, and M. F. Randolph, "Three-dimensional large deformation finite-element analysis of plate anchors in uniform clay," Journal of Geotechnical and Geoenvironmental Engineering, vol. 136, no. 2, pp. 355-365, 2010.

[26] M. Yang, C. P. Aubeny, and J. D. Murff, "Behavior of suction embedded plate anchors during keying process," Journal of Geotechnical and Geoenvironmental Engineering, vol. 138, no. 2, pp. 174-183, 2012.

[27] J. Liu, L. Lu, and L. Yu, "Keying behavior of suction embedded plate anchors with flap in clay," Ocean Engineering, vol. 131, pp. 231-243, 2017.

[28] T. M. Evans and N. Zhang, "Three-dimensional simulations of plate anchor pullout in granular materials," International Journal of Geomechanics, vol. 19, no. 4, article 04019004, 2019.

[29] S. P. Singh and S. V. Ramaswamy, "Response of plate anchors to sustained-cyclic loading," Indian Geotechnical Journal, vol. 32, no. 2, pp. 161-172, 2002.

[30] S. P. Singh and S. V. Ramaswamy, "Influence of frequency on the behaviour of plate anchors subjected to cyclic loading," Marine Georesources \& Geotechnology, vol. 26, no. 1, pp. 36-50, 2008.

[31] M. Rahimi, S. N. Moghaddas Tafreshi, B. Leshchinsky, and A. R. Dawson, "Experimental and numerical investigation of the uplift capacity of plate anchors in geocell-reinforced sand," Geotextiles and Geomembranes, vol. 46, no. 6, pp. 801-816, 2018.

[32] H.-L. Ma, M. Zhou, Y. Hu, and M. S. Hossain, "Interpretation of layer boundaries and shear strengths for soft-stiff-soft clays using CPT data: LDFE analyses," Journal of Geotechnical and Geoenviroment Engineering, vol. 142, no. 1, article 04015055, 2016.

[33] H. Hibbitt, B. Karlsson, and P. Sorensen, Abaqus Analysis User's Manual Version 2016, Dassault Systèmes Simulia Corp., Providence, RI, USA, 2016.

[34] J.-B. Zheng, M. S. Hossain, and D. Wang, "Numerical modeling of spudcan deep penetration in three-layer clays," International Journal of Geomechanics, vol. 15, no. 6, article 04014089, 2015. 


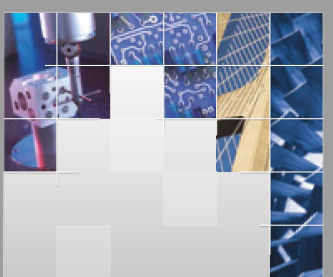

\section{Enfincering}
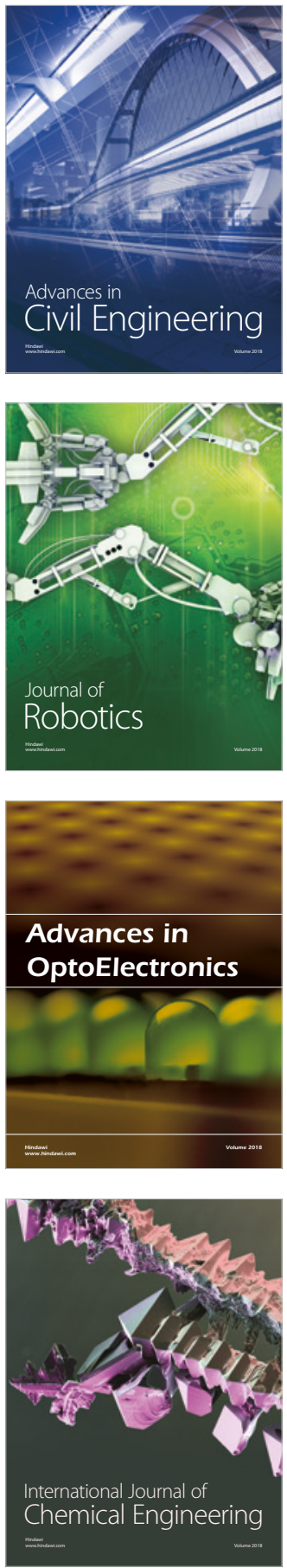

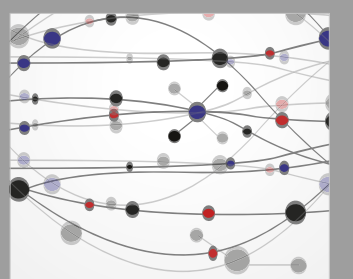

\section{Rotating \\ Machinery}

The Scientific World Journal

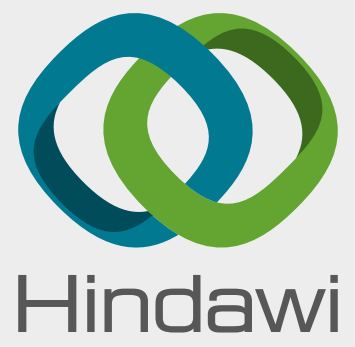

Submit your manuscripts at

www.hindawi.com
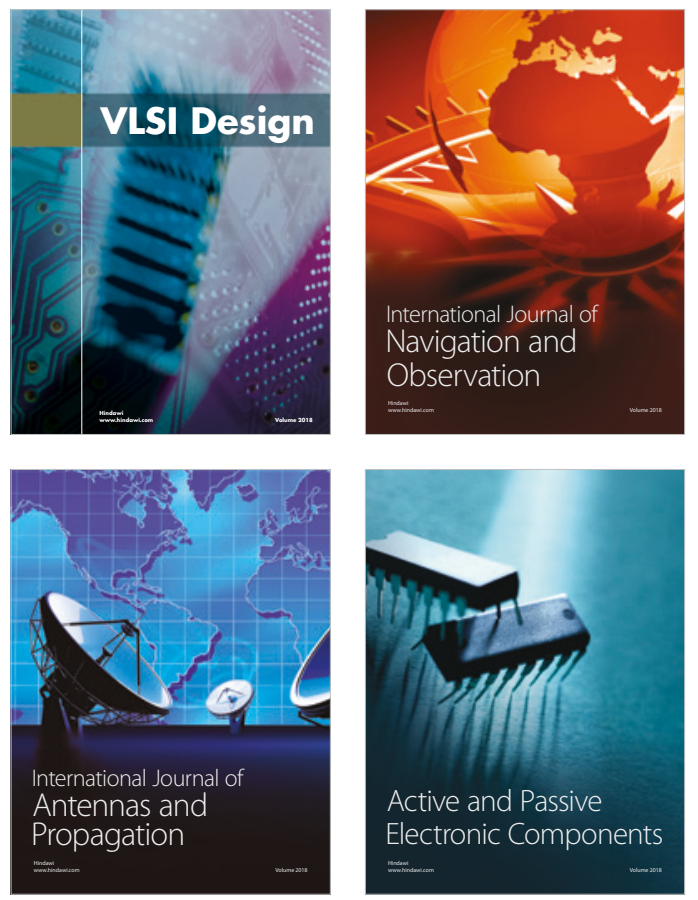
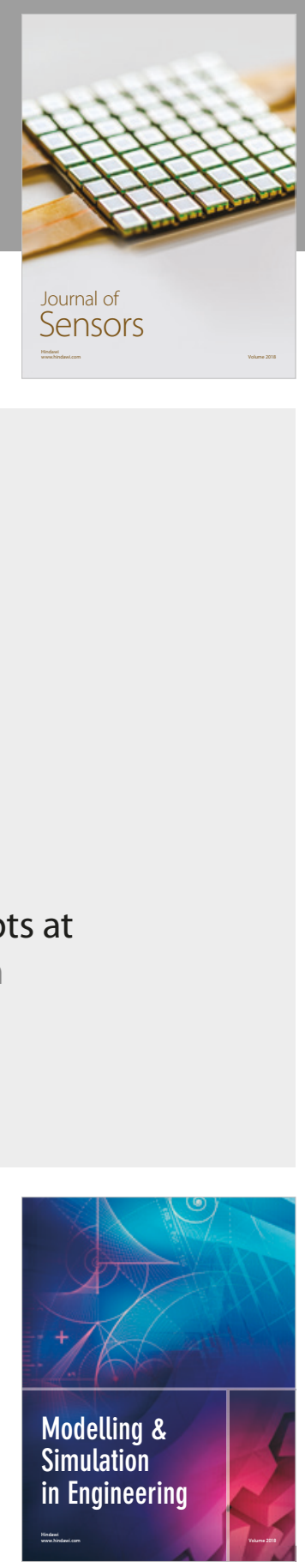

\section{Advances \\ Multimedia}
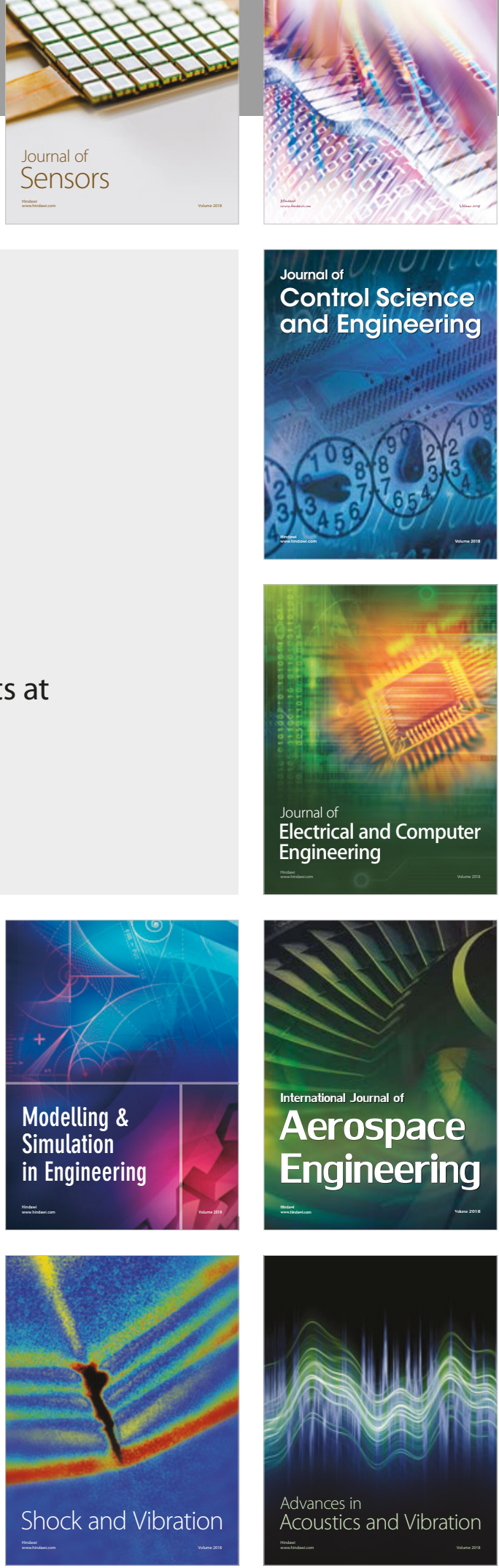